\title{
Analysis of gratings induced in azo-dye doped liquid crystals
}

\author{
L. Frey $^{\mathrm{a}, *}$, M. Kaczmarek ${ }^{\mathrm{b}}, \mathrm{J}-\mathrm{M}$. Jonathan ${ }^{\mathrm{a}}$, G. Roosen ${ }^{\mathrm{a}}$ \\ a Laboratoire Charles Fabry de l'Institut d'Optique, Unité Mixte de Recherche du CNRS no 8501, Centre Scientifique, \\ BP. 147, Bât. 503, Orsay Cedex 91403, France \\ ${ }^{\mathrm{b}}$ School of Physics, University of Exeter, Exeter EX4 4QL, UK
}

\begin{abstract}
We investigate the formation of photoinduced gratings in a homeotropic film of nematic liquid crystal doped with methyl red. Using a grating translation technique, we determine the time evolution of the amplitudes and phase shifts of both index and absorption modulations. With low writing intensities, a fast grating caused by photoisomerization of the dye competes with a slower grating, originating from a reorientation of the director. This slower grating only appears if the light polarization has a nonzero projection on the director, i.e. at oblique incidence. The grating decays in the dark. We suggest that its formation may be explained by a light-induced dielectric torque, rather than a photorefractive effect. With high intensities, an even slower grating is formed at normal incidence. We stipulate that it originates from a change in the anchoring parameters caused by the adsorption of photoisomerized dyes on the surfaces. The resulting reorientation of the director is permanent. (c) 2001 Elsevier Science B.V. All rights reserved.
\end{abstract}

Keywords: Doped liquid crystal; Gratings; Photoisomerization; Reorientation

\section{Introduction}

Nematic liquid crystals are well known for their linear and nonlinear optical properties [1]. Their strong birefringence is used to generate modulation of refractive index by molecular reorientation, with an electric field, or under the action of light. In dye-doped nematics, different phenomena may cause reorientation induced by polarized light: the torque induced by the anisotropy of the optical permittivity, and enhanced by some dopants [2]; photoisomerization and reorientation of the azodyes followed by the reorientation of the liquid crystal [3]; photoadsorption of the azo-dyes onto

\footnotetext{
${ }^{*}$ Corresponding author. Tel.: +33-1-6935-8738; fax: 33-16935-8700.

E-mail address: laurent.frey@iota.u-psud.fr (L. Frey).
}

the surface of the sample, changing the anchoring conditions [4]; photorefractive-like effect [5].

In particular, the nematic liquid crystal $5 \mathrm{CB}$ with Methyl Red (MR) was found to exhibit an extraordinarily high Kerr coefficient [6]. In $6 \mu \mathrm{m}$ homeotropic films, gratings with diffraction efficiencies up to $30 \%$ were written with intensities of a few $\mathrm{W} / \mathrm{cm}^{2}$ at $488 \mathrm{~nm}$, with a response time of a few seconds. The grating pitch was of the order of the sample thickness. Gratings could be written with beams illuminating a sample around normal incidence, but were more efficient at oblique incidence. The polarization dependence of the diffraction of a $\mathrm{He}-\mathrm{Ne}$ probe beam showed that the grating originates from a reorientation of the director in the plane of incidence. This reorientation was first attributed to a photorefractive-like effect.

However, no beam-coupling was observed, as would be expected from an index grating phase- 
shifted with respect to the interference writing pattern [6]. Moreover, the effective electro-optic coefficient vanishes with symmetrically incident beams, in a centrosymmetric material. Although several aspects of light-induced director reorientation in dye-doped liquid crystals are understood, the microscopic origin of the gratings is still unclear $[7,8]$.

Here we report experiments on a similar sample of homeotropic E7 doped with MR and $12 \mu \mathrm{m}$ thick. We first investigate single beam spectroscopic measurements followed by experiments on writing gratings. We also discuss different physical phenomena that can be involved in grating formation and the importance of illumination conditions.

\section{Dichroism}

Dichroism measurements provide information on the orientation of the MR. We thus measure absorption spectra with a probe beam in either TM or TE polarization, incident at $45^{\circ}$ from the sample normal. MR is an azo-dye and under the action of light, it photoisomerizes from the trans to the cis form [9]. The spectra show the absorption band of the MR in the trans form [4]. The sample is dichroic, indicating that the MR are roughly aligned along the director, perpendicular to the surfaces. Using the usual oriented gas model [10], we deduce the average value of the second order Legendre polynomial, i.e. the order parameter $A_{2}=0.12$.

Then we illuminate the sample with a $800 \mathrm{~mW} / \mathrm{cm}^{2}$ pump laser beam at $532 \mathrm{~nm}$ (frequency doubled Nd:YAG). After a few seconds, the absorbance decreases, in the same proportions for the TM $(-19 \%)$ and TE $(-16 \%)$ spectra. This change is reversible. The same results are obtained for both polarizations and the direction of the pump beam, showing that the MR photoisomerize but does not reorient. This behavior is different from a number of azo-dye doped polymers [11].

\section{Gratings written with low intensities}

Gratings are induced by two beams from the same laser, each $800 \mathrm{~mW} / \mathrm{cm}^{2}$, crossing at an angle of $2.2^{\circ}$, with angles of incidence approximately $45^{\circ}$. The grating pitch is then approximately $18 \mu \mathrm{m}$. To write and analyze the gratings, we use a grating translation technique [12], which provides the amplitudes and phase shifts of the index and absorption modulations, in real time. The setup has to be stable to a high degree to get rid of unwanted drifts of the interference pattern.

With TM polarizations, a mixed index and absorption grating of negative sign (phase shift $\pi$ ) forms in a few seconds (Fig. 1). The magnitude of refractive index and absorption decrease in the bright fringes, probably because of photoisomerization of the MR. This is consistent with the fast decrease of the absorption spectra under pump illumination. Then the grating tends to disappear, either by a saturation of the grating [13], or by a competition with the slower, positive grating (phase shift 0 ) which begins to appear and dominates at longer times. It is mostly a pure index grating.

With TE polarizations, the fast grating has approximately the same or slightly lower efficiency, but the slow grating is strongly diminished.

This slower phase grating probably originates from a reorientation of the liquid crystal director towards the polarization direction, in the bright fringes. From the diffraction efficiency and the indices of refraction $n_{\mathrm{e}}=1.738$ and $n_{0}=1.537$ at $546 \mathrm{~nm}$, we deduce an average value of the director rotation of about $1^{\circ}$. Anchoring forces hinder a stronger reorientation.

The local nature of the grating seems inconsistent with a photorefractive effect. In order to confirm this, we try to write a grating with the sample tilted of $45^{\circ}$ out of the plane of the writing beams, as opposed to the experiment of Fig. 1 where it is tilted in the plane of the beams. The polarization is still TM, i.e. in the plane of incidence. We observe the same grating. Therefore, we can conclude that the necessary condition to write the grating is the existence of a projection of the polarization onto the director, and not onto the direction of the intensity gradient.

We also exclude a photoadsorption effect [4]. The grating would be permanent, and the index grating accompanied by an adsorption grating. 

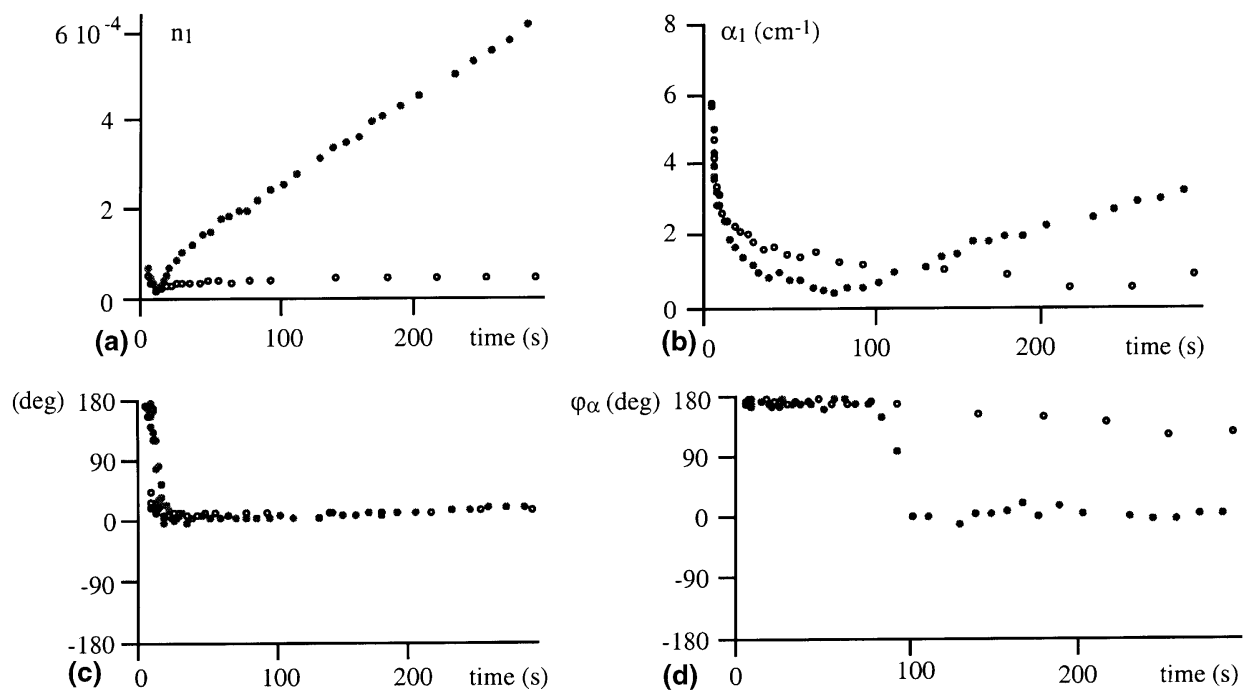

Fig. 1. Time dependence of the gratings induced at $532 \mathrm{~nm}$ by two $800 \mathrm{~mW} / \mathrm{cm}^{2}$ beams intersecting at $2.2^{\circ}$ at oblique incidence. Full disks: TM polarization; empty disks: TE polarization. (a) Modulation $n_{1}$ of the refractive index; (b) modulation $\alpha_{1}$ of the absorption coefficient; (c) phase shift $\varphi_{n}$ of the index grating; (d) phase shift $\varphi_{\alpha}$ of the absorption grating.

The observations are consistent with the results and interpretation provided by other groups [2]. The origin of the reorientation of the director could be the light-induced torque originating from the anisotropy of the optical premittivity, and enhanced by the MR. This would explain why the slow grating vanishes with TE polarization, as well as for normal incidence, as the polarization is then perpendicular to the director. Under these conditions the reorientation should occur only above a certain intensity threshold.

The grating seems to be different from the one observed in [6], where the response time is much faster and the grating is still observed at normal incidence, although less efficient. However, the response time decreases by a factor of 10 when we use an argon laser to write the grating, with a 514 $\mathrm{nm}$ wavelength being closer to the center of the MR absorption band.

\section{Gratings written with high intensities}

With higher intensities $\left(6 \mathrm{~W} / \mathrm{cm}^{2}\right)$, we can write gratings even at normal incidence. They may only be read by a beam polarized in the same direction as the writing beam. A diffusion background progressively appears, as diffraction grows. These gratings contain both index and absorption modulations of positive sign (see Fig. 2 for the index
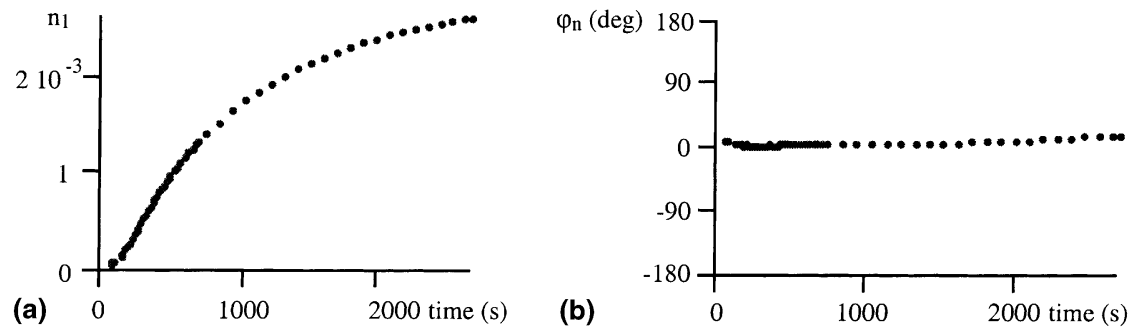

Fig. 2. Time dependence of the gratings written at normal incidence, $532 \mathrm{~nm}, 6 \mathrm{~mW} / \mathrm{cm}^{2}$. (a) Modulation $n_{1}$ of the refractive index; (b) phase shift $\varphi_{n}$ of the index grating. 
grating). The response time is slow compared to this measured at oblique incidence.

The gratings are permanent, which may be explained by a change of the anchoring conditions. An interpretation involving adsorption of the MR on the surfaces, parallel to the polarization, and subsequent reorientation of the liquid crystal, seems to be the most likely mechanism [4]. The large index grating observed here is caused by a reorientation of the director of $\delta \theta=8^{\circ}$ after $2500 \mathrm{~s}$. We suggest that the absorption grating originates from the microassembly of dye molecules on the surface [14].

\section{Conclusion}

We analyzed the formation of gratings in nematic E7 doped with MR. Our results suggest that a photorefractive-like effect is not responsible for their build-up. We suggest that the intensity of illuminating light plays a crucial role in determining the underlying mechanism for gratings formation. At low intensities, light-induced torque originating from the anisotropy of the optical permittivity, increased by the dopant, may be responsible for the index variation. At high intensities, the permanent gratings may be generated by the adsorption of the phototransformed dopant onto the surfaces.

\section{References}

[1] I.C. Khoo, Liquid Crystals: Physical Properties and Nonlinear Optical Phenomena, Wiley/Interscience, New York, NY, 1995.

[2] I.C. Khoo, IEEE J. Quantum Electron. 29 (1993) 1444.

[3] T.Y. Marusii, Y.A. Reznikov, S.S. Slussarenko, Mol. Mater. 6 (1996) 163.

[4] D. Voloschenko, A. Khyzhnyak, Y. Reznikov, V. Reshetnyak, Jpn. J. Appl. Phys. 34 (1995) 566.

[5] I.C. Khoo, IEEE J. Quantum Electron. 32 (1996) 525.

[6] I.C. Khoo, S. Slussarenko, B.D. Guenther, M.-Y. Shih, P. Chen, W.V. Wood, Opt. Lett. 23 (1998) 253.

[7] I.C. Khoo, M.-Y. Shih, M.V. Wood, B.D. Guenther, P.H. Chen, F. Simoni, S.S. Slussarenko, O. Francesangeli, L. Lucchetti, Proc. IEEE 87 (1999) 1897.

[8] F. Simoni, O. Francescangeli, J. Phys.: Condens. Mater. 11 (1999) R439.

[9] Y. Shi, W.H. Steier, L. Yu, M. Chen, L.R. Dalton, Appl. Phys. Lett. 58 (1991) 1131.

[10] K.D. Singer, M.G. Kuzyk, J.E. Sohn, J. Opt. Soc. Am. B 4 (1987) 968.

[11] T. Todorov, L. Nikolova, N. Tomova, Appl. Opt. 23 (1984) 4309.

[12] L. Frey, J.-M. Jonathan, A. Villing, G. Roosen, Opt. Commun. 165 (1999) 153.

[13] L. Frey, B. Darracq, F. Chaput, K. Lahlil, J.-M. Jonathan, G. Roosen, J.-P. Boilot, Y. Lévy, Opt. Commun. 173 (2000) 11.

[14] D. Voloschenko, O.D. Lavrentovitch, J. Appl. Phys. 86 (1999) 4843. 